\title{
Smart Grid Dispatching Optimization for System Resilience Improvement
}

\author{
Li Liao $\mathbb{D D}^{1,2}$ and Chengjun Ji ${ }^{1}$ \\ ${ }^{1}$ School of Business Administration, Liaoning Technical University, Huludao, Liaoning 125105, China \\ ${ }^{2}$ School of Economics and Management, Zhejiang University of Science and Technology, Hangzhou 310013, China \\ Correspondence should be addressed to Li Liao; liaoli@zust.edu.cn
}

Received 28 September 2020; Revised 26 October 2020; Accepted 29 October 2020; Published 6 November 2020

Academic Editor: Wei Wang

Copyright (c) $2020 \mathrm{Li}$ Liao and Chengjun Ji. This is an open access article distributed under the Creative Commons Attribution License, which permits unrestricted use, distribution, and reproduction in any medium, provided the original work is properly cited.

\begin{abstract}
A large number of modern communication technologies and sensing technologies are incorporated into the smart grid, which makes its structure unique. The centralized optimized dispatch method of traditional power grids is difficult to achieve effective dispatch of smart grids. Based on the analysis of power generation plan and maintenance plan optimization model, this paper establishes a smart grid power generation and maintenance collaborative optimization model with distributed renewable energy. The objective function of this collaborative optimization problem is the operating cost of conventional units, the cost of wind power generation, and the cost of overhauling units; the constraints considered mainly include system constraints and overhaul constraints. The solution method of combinatorial optimization is analyzed, and the genetic optimization algorithm adopted in this paper is selected and discussed. According to the characteristics of the system, various loads are modeled, and power supply constraints are considered. By establishing an effective objective function, the adjustable load scheduling problem is transformed into a solvable optimal control problem. Taking into account the uncertain factors in the system, the advantage of the real-time control system is that it can realize the dynamic update scheduling of the load, so it is more in line with the requirements of the actual system. The real-time algorithm proposed in the paper is based on a distributed control strategy, which can not only realize dynamic compensation for random fluctuations in renewable energy power generation but also satisfy the load curve optimization under the premise of making full use of power supply resources. In addition, simulation experiments compare the load dispatching capabilities of the proposed algorithm with the existing algorithms, thereby verifying the performance of the proposed method.
\end{abstract}

\section{Introduction}

The rapid development of the global economy has increasingly demanded the quality and quantity of electric energy. However, fossil fuels are facing exhaustion, and as environmental pollution becomes more and more serious, all sectors of society urgently need technology to improve energy efficiency and power safety and stability [1]. In this case, smart grid technology came into being. And this technology has caused extensive discussion and focused research in recent years. Due to the widespread application of smart grids, enterprises can operate efficiently and reliably, making it possible to significantly reduce the operating costs of enterprises [2,3]. By optimizing the management from production to transportation to retail, the enterprise management is made intelligent, so as to achieve the goals of saving electricity, increasing the utilization rate of renewable energy, supporting the access of hybrid vehicles, and making home appliances intelligent [4]. It is the ultimate goal of the grid company to make the power grid operate safely and reliably, and the construction cost becomes more economical [5].

After entering the 21st century, country's power supply situation has become increasingly tense. During the power supply shortage period, especially during the peak summer period, about 27 provincial power grids across the country have been shut down and limited, and the problem called "power shortage" is still widespread. At present, there is a large peak-valley difference in the electric load curve, and the 
peak load is still increasing, resulting in an increasing peakvalley difference. The direct consequence of this situation is the occurrence of insufficient power supply. If we want to completely change this kind of phenomenon, we can only increase the power supply during peak periods. However, in a short period of time, the most effective solution at present is to carry out reasonable load dispatching, by making full use of the available resources in the power system for full allocation and dispatching, so as to reduce the peak load, increase the trough value, and reduce the difference between the peaks.

Smart grid multiagent systems respond to these challenges through effective science and technology, and smart grid distributed dispatching strategies are increasingly being valued by power system researchers [6]. In the actual environment, when a multiagent system is running, each agent will have a time delay due to the distance between each agent when receiving information sent by other agents [7]. The safe and stable operation of the power system needs to consider the influence of the communication system. The time delay between smart grid multiagent systems often affects the dynamic performance of the multiagent system, which may reduce the convergence speed of the system, and more seriously, it may make the system unstable [8]. In the future power system, researchers have discussed plug and play as an effective way to connect resources to the grid [9]. Similar to computer systems, plug and play in power systems means that a plug-in can be placed anywhere in the electrical system without reset control [10]. The plug-and-play interface will include a communication interface, so when a new device is added to the substation, it automatically reports data to the control center, such as device parameters and device interconnection information $[11,12]$. Therefore, the control center must have high-bandwidth communication facilities to collect all the information in the system and require high connectivity in the communication topology of the system, which increases the investment in the communication topology and puts high demands on the computing power of the control center [13]. Due to the plug-and-play feature, different devices frequently access and exit, which makes the topology of the system change [14]. In order to control this kind of system, distributed optimization is more suitable for solving the changing communication topology and adapting to the requirements of plug and play. The distributed algorithm has higher robustness and scalability and can better adapt to renewable energy power generation. Relevant scholars have proposed a robust load and energy management framework for a sustainable data center, which solves the problem of optimal workload and power management in a cloud network composed of multiple geographically distributed mapping nodes and data centers [15]. In order to deal with the uncertainty of renewable energy power generation, the author uses a polygonal area constructed with linear constraints such as the maximum and minimum solar power generation as uncertainty set and constructs resource allocation and task allocation as a robust optimization problem [16]. It minimizes the net cost in the worst case of data center operation constraints. Researchers regard the interaction between the dynamic electricity price of the smart grid and the energy consumption load of the data center as a game problem [17].
In the first stage, the smart grid uses dynamic pricing to change the energy consumption of the data center to balance the power load. In the second stage, the data center minimizes the total electricity bill by managing the load distribution in response to changes in electricity prices. Related scholars have proposed a cost minimization problem, which is to intelligently dispatch microgrid power generation equipped with unstable renewable energy and cogeneration generators [18]. In order to deal with the uncertainty of net demand and thermal demand, the author proposes a novel and flexible uncertainty model, which solves the main problem of minimizing cost through the branch and bound method [19]. Relevant scholars considered the multiobjective optimization problem in the task scheduling of the data center, established a four-objective framework, optimized the utilization rate of renewable energy, task completion time, total energy consumption, and task processing rate at the same time, and proposed an enhanced multiobjective coevolutionary algorithm to deal with this problem [20].

In the context of the rapid development of smart grids and the large-scale development and utilization of renewable energy, this article considers the impact of large-scale wind power grid connection on the preparation of dispatch plans and establishes a smart grid power generation plan and maintenance with distributed renewable energy. We utilize the distribution function of wind power output to transform wind power uncertainty into a deterministic model and use intelligent optimization algorithm (genetic algorithm) to solve the model, and the optimized dispatching plan will reserve enough peaking space for wind power fluctuations. The limited consumption of wind power will bring considerable economic benefits. An optimal control algorithm is proposed in this paper, which realizes the tracking of the load curve to the power supply curve by minimizing the variance of the difference between the load and the power supply. When compared with traditional control strategy algorithms, simulation experiments verify the effectiveness of the algorithm in this paper. Aiming at the real-time distributed system, an adjustable load dispatching strategy with power supply constraints is proposed. This strategy can compensate for fluctuations in the power generation process and realize real-time tracking of the load curve to the power supply curve. By predicting related variables, the adjustable load can be randomly connected to the grid, concepts such as virtual load are introduced, and then real-time control methods are proposed.

The rest of this paper is organized as follows. Section 2 analyzes the resilience of smart grid systems. Section 3 establishes a coordinated dispatch optimization model for smart grid generation and maintenance. In Section 4, a simulation experiment is carried out and the experimental results are analyzed. Section 5 summarizes the full text.

\section{Analysis of Smart Grid System Resilience}

2.1. Smart Grid Dispatching Technology. Smart grid covers a wide range, including basic systems for power transmission and distribution, and integrates many high-tech technologies such as artificial intelligence technology, digital 
technology, sensor technology, information technology, and communication technology. As environmental issues become increasingly prominent, how to build a new type of power grid that is environmentally friendly, efficient, reliable, and highly intelligent has become a common problem for everyone. The establishment of a smart grid can integrate, analyze, and utilize the huge information of the entire grid and enable the grid to form a customer service bus through information induction calculations, maximize the analysis and utilization of information and data, ensure the stability of the entire system, and make the operation and management level more efficient.

One of the characteristics of electric energy is that it cannot be stored. The power plants scattered over a large area generate electricity, which is boosted by high-voltage substations, and then passed through high-voltage transmission lines, step-down substations, and smart grids to users. Grid dispatch is an important part of the smart grid. Smart dispatch is an important manifestation of the technical and application level of the smart grid. It is the key to quickly improve the power grid's ability to accept clean energy and is the only way to build a smart grid. Figure 1 depicts the relationship between dispatch and other systems in the entire smart grid.

\subsection{Analysis of Smart Grid Failure Rate}

2.2.1. Failure Rate Analysis Based on Structural Reliability Theory. The force load covers factors such as the size of the force and the point of action; the strength of its own structure is related to the material and size of the components used. The influence of extreme weather on power grid components on the failure rate can be analyzed using structural reliability theory.

When the load effect on the wires and poles exceeds the strength of the components, the components will be damaged, and the components of the wires and poles obey a certain probability distribution. Random variable of wire strength: overhead wires are generally made of steel-cored aluminum stranded wires, which will break under the action of external force. Therefore, the strength of the wires mainly considers their tensile strength, that is, the maximum comprehensive stress that they can withstand when the wire is broken. The IEC60826 standard points out that the tensile strength of the wire material obeys a normal distribution, and its probability density function can be expressed as

$$
f_{R}(\sigma)=\frac{1}{\sqrt{2 \pi}} \delta_{l} \exp \left(\frac{u_{l}-\sigma}{\sqrt{2} \delta_{l}}\right)^{2} .
$$

In the formula, $u_{l}$ and $\delta_{l}$ are the mean and standard deviation of the tensile strength of the wire, respectively.

The strength of the electric pole of the overhead conductor in the smart grid is also uncertain due to the manufacturing and construction errors and other reasons. The strength of the electric pole component mainly focuses on its bending strength, that is, the maximum bending moment that it can bear. The bending strength obeys the normal distribution, and its probability density function can be expressed as

$$
f_{R}\left(M_{p}\right)=\frac{1}{\sqrt{2 \pi} \delta_{p}} \exp \left(\frac{u_{p}-M_{p}}{\sqrt{2} \delta_{p}}\right)^{2} .
$$

In the formula, the mean value $\mu p$ and standard deviation $\delta p$ of bending strength can be obtained through actual operating experience.

\subsubsection{Analysis of Failure Rate Based on Short-Circuit Trip} Rate Model. Different from extreme weather such as typhoons and rainstorms, mechanical loads affect the failure rate of distribution lines. Thunderstorms will cause overhead distribution lines to trip faults. Some of the trips are transient faults that can be restored by automatic reclosing, and the other is permanent. Sexual faults may be caused by insulators falling off and wires broken. The probability of tripping faults is related to the lightning density, lightning resistance level, dielectric strength, and lightning current amplitude.

When the lightning strikes the ground, the hit point is uncertain. It depends on which object the first end of the discharge channel enters first. It falls within the lead distance $R_{d}$ and causes a direct lightning trip and falls within the ground strike distance $R_{e}$.

The calculation formulas for the distance between the wire and the ground are

$$
\begin{aligned}
& R_{d}=0.64 \cdot h_{e}^{0.55} \cdot I_{i}^{0.73}, \\
& R_{e}=R_{d} \cdot\left[0.34+0.163 \cdot \ln \left(42-h_{e}\right)\right] .
\end{aligned}
$$

In the formula, $h_{e}$ is the average height of the wire. The height of the intersection of the ground exposure arc and the wire exposure arc is equal to the lightning current $I_{0}$ corresponding to $h_{e}$ :

$$
I_{0}=\left(\frac{\cos \theta \cdot h^{0.3}}{0.64 \cdot(0.34-\sin \theta+0.166 \cdot \ln (42-h))}\right)^{(1 / 0.73)} .
$$

\subsection{Analysis of Fault Scenarios considering the Feeder Area.} To perform smart grid resilience analysis, it is necessary to understand the load loss caused by extreme weather. Therefore, it is necessary to determine the power outage range according to the network structure and the location of the faulty component and calculate the lack of power supply. It is necessary to analyze the lack of power supply in each fault scenario and determine the power outage range and power outage time according to the location of the faulty component, the network structure, and the switch position.

During the planning and design of the power distribution system, it is generally required to meet the $N$ - 1 safety criterion, but the open-loop operation mode should be adopted during operation. When the smart grid component fails, the contact switch will act, and some loads can be restored to supply power; some loads are isolated from the 


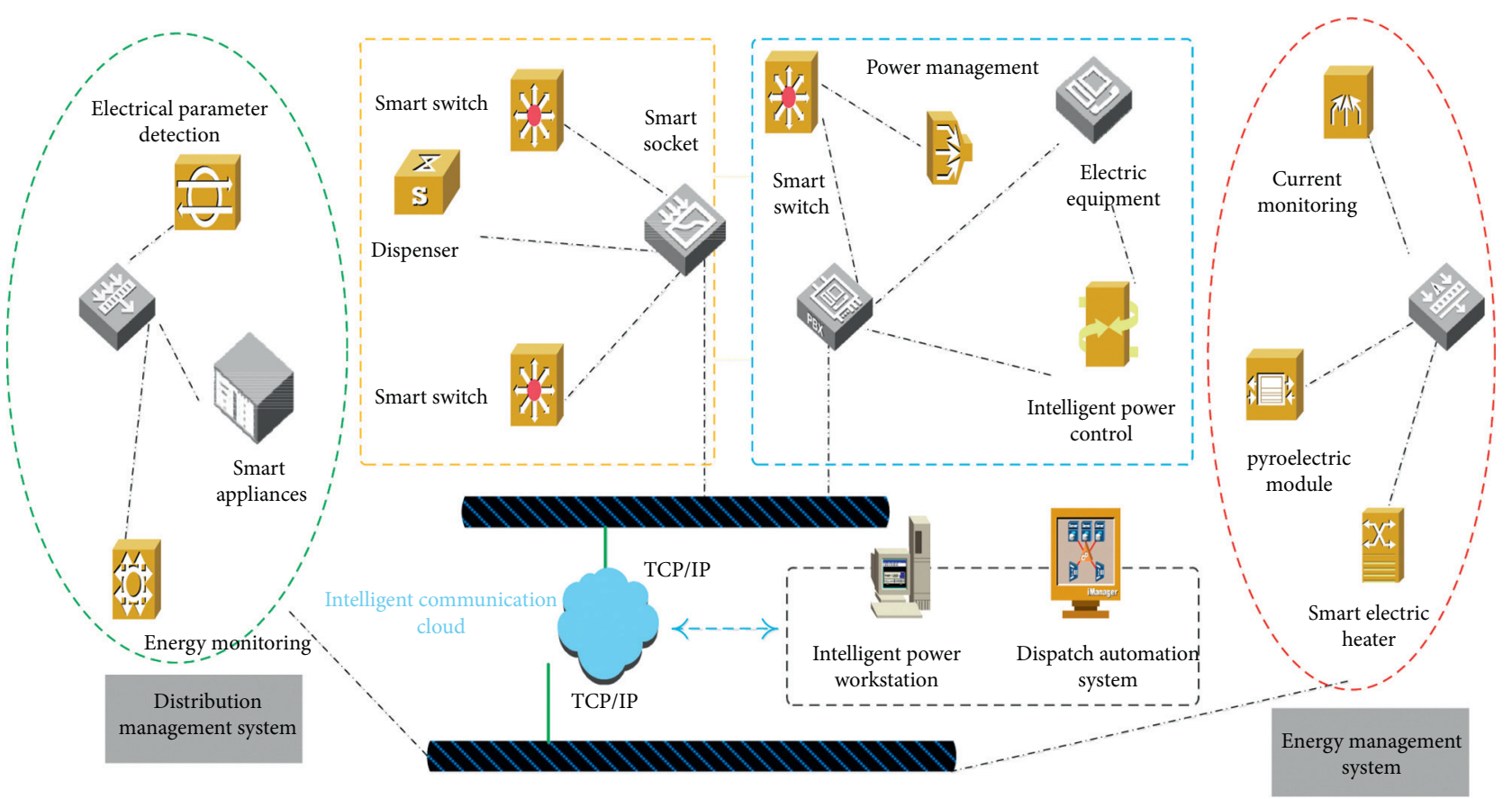

FIGURE 1: The relationship between the smart grid dispatching system and other systems.

fault area and continue to be powered by the superior power supply; the other part of the load cannot be separated from the fault area, and the power supply will be restored after the component is repaired. The range of load power outage in the fault scenario is related to the network structure; the power outage time is related to the segmentation formed by the switching elements.

When a component on the feeder fails, the fault can be isolated by a switch, and part of the load can be restored to supply power. Therefore, the fault range analysis in a fault scenario mainly depends on the position of the switch component, and the load in the same segment has the same power failure state. For multiple faults, when the fault conditions of other components are the same, all components in the same segment have the same impact after failure. The concept of feeder area can simplify the analysis of the impact range of the power distribution system fault scenario. The feeder area refers to a collection of components that have a common and contain only one inlet switch. The failure of a component will cause other components in the same feeder area to lose power. Therefore, the power loss of the components in the feeder section can be analyzed in the unit of feeder area, thereby improving the efficiency and speed of power shortage calculation in different scenarios.

Under extreme weather conditions, the smart grid fails on a large scale and a large-scale blackout occurs. After the extreme weather passes the border, the smart grid gradually returns to its original normal operating state. Figure 2 is a schematic diagram of the system function curve of the smart grid during the entire process of extreme weather.

According to the location, failure time, and repair sequence of the faulty components in each scenario, we analyze the power loss of each node load point from the consequences

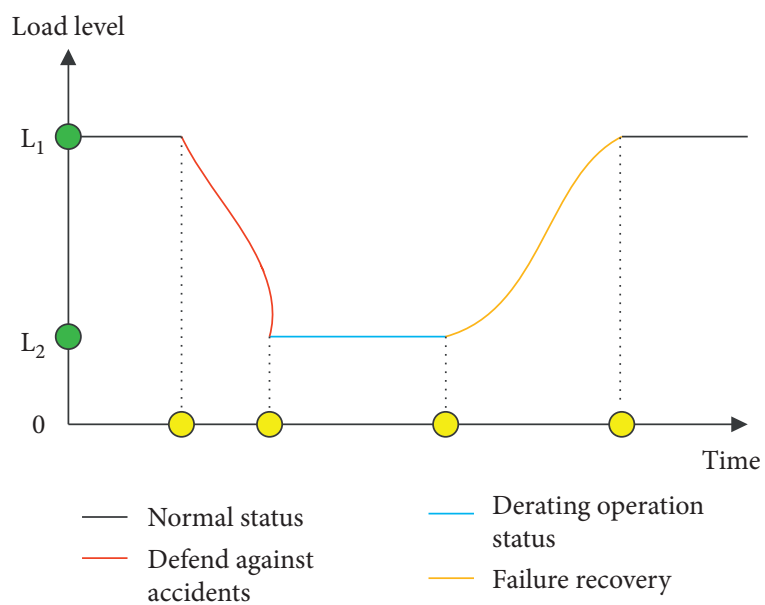

Figure 2: Schematic diagram of the system function curve of the smart grid under extreme weather.

of different components failure and obtain the system functions from the occurrence of extreme weather to the restoration of normal operation and then calculate the missing area of the load curve of the entire system in the fault scenario.

2.4. Smart Grid Resilience Assessment Model. The main impact of extreme weather on the smart grid is a significant increase in the component failure rate, resulting in an increase in the probability of large-scale multiple failures. In this scenario, the failure and recovery process is very complicated. There are many smart grid components, and there are a huge number of multiple failure scenarios composed of different failure components. Therefore, it is necessary to analyze the 
failure scenarios that may be caused by extreme weather according to the possibility and uncertainty of the scenarios and the failure rate of the overhead lines. The system information entropy method is a method of selecting reasonable system state scenarios based on the probability of a single event. Entropy represents the degree of uncertainty of the system. Smart grid is an uncertain system that may fail at every moment, and its entropy value $W$ is

$$
W=-\sum_{i \in \Omega_{B}} \log _{2}\left(z_{i, t} \cdot p_{i, t}\right), \quad t \longrightarrow T .
$$

Among them, $T$ represents the time taken by the typhoon to traverse the smart grid area; $\Omega_{B}$ represents the smart grid line set; $z_{i, t}$ represents whether line $i$ happens to be faulty at time $t$; and 1 is a fault. Each toughness analysis scene corresponds to a $z_{i, t}$ vector, which corresponds to the entropy value of the system in that scene.

From the perspective of the uncertainty of the failure scenario, the value of $z_{i, t}$ should obey the distribution of the failure rate. The higher the failure rate of a certain line, the greater the probability of the occurrence of such an uncertain event of the line failure. For example, if the failure rate of line $i$ is 0 , the uncertainty of the component failure event will be infinite, and there must be $z_{i}$ in all scenarios, $t=0$. Therefore, judging from the possibility of the actual scenario, the reasonable toughness analysis scenario $W$ cannot be too big or too small to satisfy:

$$
\left\{\begin{array}{l}
\sum_{t} z_{i, t}<1 \\
W_{\max }>-\sum_{i \in \Omega_{B}} \log _{2}\left(z_{i, t} \cdot p_{i, t}\right)>W_{\min } .
\end{array}\right.
$$

The fault scenarios selected to meet the conditions have the characteristics of a greater probability of occurrence and serious consequences of the fault and constitute a typical fault scenario in the smart grid resilience analysis.

This paper uses the missing area of system functions under extreme weather to reflect the resilience of the smart grid and uses the occurrence probability of failure scenarios and the corresponding missing area of the load curve to calculate the smart grid resilience index.

$$
A R=E \frac{\int_{0}^{T_{0}} T L(t)-R E S_{n}}{\int_{0}^{T_{0}} T L(t) \mathrm{d} t} \cdot \sum_{n=0}^{N-1} \lambda_{n} \cdot \operatorname{Im}_{n} .
$$

In the formula, $\lambda_{n}$ is the probability of occurrence of scenario $n ; N$ is the number of selected failure scenarios; $\operatorname{Im}_{n}$ is the degree of lack of power supply in scenario $n ; T_{0}$ represents the time that the smart grid is affected by extreme weather, including the time it takes for the typhoon to cross the smart grid. Since the time required for fault recovery in each scenario is different, $T_{0}$ should be much greater than the corresponding $t 4$ in each scenario; $L(t)$ represents the actual load when a large-scale fault occurs due to extreme weather; $T L(t)$ represents the target load curve when the system is running without failure; $R E S_{n}$ represents the area between the actual curve and the target curve, which is the missing area of the load curve.

\section{Smart Grid Coordinated Dispatch Optimization Modeling}

3.1. Collaborative Optimization Model. The modes of power dispatch are usually divided into economic dispatch mode, energy-saving power generation dispatch mode, and lowcarbon power dispatch mode. In different dispatch modes, the objective function used in the optimization of generation plan is different. Under normal circumstances, economic dispatch mainly considers the operating cost of the system; energy-saving optimization dispatch mainly considers the energy consumption of the system and the emission of pollutants; and low-carbon dispatch mainly considers the emission of carbon dioxide. The schematic diagram of data verification in the power dispatch data center is shown in Figure 3.

The constraints that need to be considered in the optimization of power generation plans involve a wide range and complexity. From a comprehensive classification point of view, it mainly includes the constraints of the generator set itself, the group constraints, the system constraints, and the network constraints. Among them, the self-constraint of the generator set refers to the constraint that only involves a single unit, mainly including the upper and lower limits of output, the climbing constraint, the minimum start (stop) time constraint, and the number of start and stop constraints. The constraints of a generating unit mainly include the output constraints of the unit group and the power consumption of the unit group; the systemic constraints refer to the constraints involving all the generating units in the system, including active power balance constraints, standby constraints, and emission constraints.

The power generation cost of a traditional unit is usually fitted into a quadratic function on the output of the unit. In this article,

$$
f(X, Y)=0.5 \cdot a_{i} \cdot X^{2}+b_{i} \cdot X+c_{i} \cdot Y .
$$

Among them, the power generation cost coefficient of the $i$-th unit can be represented by $a_{i}, b_{i}$, and $c_{i}$; $X$ is the active power output of unit $i$ in period $t ; Y$ is the start and stop status of conventional unit $i$ in period $t ; 1$ means running; 0 means outage.

The power generation of wind turbines does not require fuel costs, and the fuel cost is set to 0 , but the maintenance costs and labor costs of the wind turbines need to be taken into account, so the cost of wind power generation in this article can be expressed as

$$
g\left(X_{k, t}^{W}\right)=\theta \cdot X_{k, t}^{W} .
$$

Among them, $\theta$ is the maintenance cost coefficient of wind turbine $k$.

3.2. Combinatorial Optimization Algorithm. Similar to the power generation plan and maintenance plan, the collaborative optimization problem is a combination optimization problem, which has the characteristics of high dimensionality, discreteness, and nonlinearity. The existing solution 


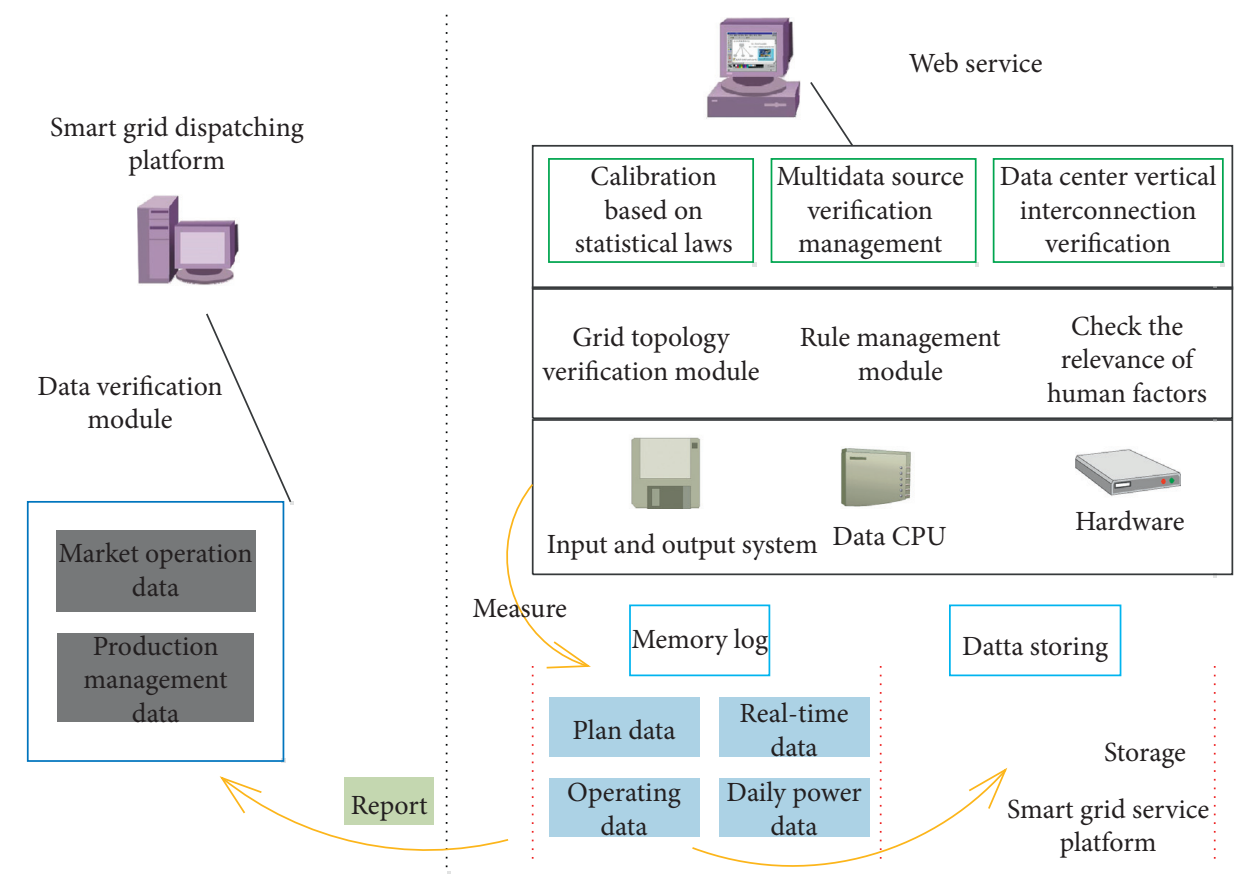

FIGURE 3: Schematic diagram of data verification in power dispatch data center.

methods can be mainly divided into heuristic algorithms, traditional mathematical optimization algorithms, and artificial intelligence optimization.

Among them, heuristic algorithms mainly include equal standby method, equal risk method, priority order method, and dynamic programming method; traditional mathematical optimization algorithms include linear programming method, 0-1 integer programming method, branch and bound method, and Lagrangian relaxation method; intelligent optimization algorithms mainly include genetic algorithm, tabu search algorithm, simulated annealing algorithm, particle swarm optimization algorithm, and neural network algorithm. Hybrid intelligent algorithm refers to the combination of two intelligent optimization algorithms, including immune-taboo search algorithm.

(1) Linear programming: Linear programming is an optimization method that has been studied earlier in operations research. It helps people to conduct scientific management so that they can make the best decisions with limited resources. The principle is to use the objective function and constraint conditions of the research problem. The linear equation is expressed and the simplex method is used to obtain the optimal solution to the problem.

(2) Mixed-integer programming: In linear programming, the decision variables of the problem can be integers or decimals and fractions. In integer programming, certain decision variables must be integers. If all variables are integers, this kind of programming problem becomes pure integers. Many problems of traditional combinatorial optimization are mixed-integer programming problems, such as the knapsack problem, the traveling salesman problem, and the unit commitment problem in the power system. A special form of mixed-integer programming is the $0-1$ problem, which can be solved mainly by enumeration.

(3) Neural network algorithm: The neural network algorithm has the characteristics of large-scale output of distributed information and good self-organization and self-learning capabilities. The algorithm research originated from human neuroscience. The artificial neural network is a dynamic system composed of a large number of interconnected neural units. It has broad application prospects in optimization design, signal output, intelligent control, and so on.

(4) Simulated annealing algorithm: The simulated annealing algorithm is designed based on the principle of solid annealing in physics. It is a random optimization method using an iterative solution strategy. The idea is to start from a higher initial temperature. As the temperature gradually decreases, there is a certain probability that it will jump out of the local solution space to find the global optimal solution. The simulated annealing algorithm is a general optimization algorithm, which is used in engineering practice. It has a wide range of applications.

(5) Particle swarm algorithm: Particle swarm optimization algorithm, also referred to as PSO algorithm, is a parallel computing method that has been studied and widely used among intelligent optimization algorithms in recent years. This method simulates the group behavior of natural organisms, based on the information-sharing mechanism, 
through the self-learning of particles and the method of learning from the best individual to achieve a rapid search for the solution space. The particle swarm algorithm is very suitable for singleobjective optimization problems or multiobjective optimization problems that are complex in solution space and inconvenient to use analytical methods.

Similar to the simulated annealing algorithm, it also starts from a random solution, finds the optimal solution through iteration, and evaluates the quality of the solution through fitness in this process. But compared with genetic algorithm, it is much simpler. There are no "cross" and "mutation" operations in genetic algorithm. It finds the global optimal solution by following the optimal value currently searched. However, in practical applications, there are still strategies such as individual elimination measures similar to genetic algorithms. Like many evolutionary algorithms, the particle swarm algorithm requires a relatively large amount of calculation, but it can still find a satisfactory optimal solution in a relatively short time when the particle dimension is small and the optimization strategy is appropriate.

(6) Dynamic planning: Dynamic programming algorithms are widely used in economic management, production scheduling, and other fields. They are mainly used to solve dynamic optimization problems divided by time. The basic idea is to decompose the problem into several stages, which can be obtained by decision-making and solution of each stage. The dynamic programming algorithm has a strong global optimal searchability, but it is easy to fall into the disaster of dimensionality in the face of large-scale planning problems. The problem solved by dynamic optimization algorithm must satisfy the optimization principle and the condition of no aftereffect.

3.3. Genetic Optimization Algorithm. Based on the principle of biological evolution, the genetic algorithm applies the process of genetic evolution and recombination to the formulation of the smart grid dispatching plan. The parameters to be solved are compiled in layers and expressed in binary or decimal codes like a gene sequence. The combination of gene sequences is called a "chromosome." The final optimization result can be obtained after several iterations.

The factors involved in the genetic algorithm include determining the coding method of the parameters, selecting the initially calculated population, designing fitness functions, genetic operations, processing algorithm control parameters, and setting constraints. The main advantages of genetic algorithm are as follows:

(1) The processing object of genetic algorithm is mainly the individual "chromosome" obtained through binary or decimal encoding, rather than the parameters themselves before encoding. The algorithm can directly manipulate structure objects such as sets, sequences, matrices, trees, graphs, chains, and tables. For example, by operating the connection matrix, the network structure and parameters are optimized.

(2) The genetic algorithm can process multiple individuals in the group, effectively avoiding the search space being trapped in a local extreme point of a single peak during point-to-point search. This makes the global search performance of genetic algorithm better.

(3) The genetic algorithm can evaluate the pros and cons of individuals only by the size of the fitness function value and perform a series of operations such as selection, crossover, and mutation based on this. The fitness function is not constrained by continuous differentiability, the coding only needs to correspond to the feasible solution space, and the domain of definition can also be set arbitrarily.

The initial population is to randomly generate the initial solution vector in the solution space through a random algorithm. The method for determining the initial population is to randomly generate $N$ initial string structure data. Each initial string structure data is an individual, and the randomly generated initial population shares $N$ individuals; these $N$ individuals together constitute a group. The iterative process starts with the genetic algorithm, takes these string structure data as the starting point, and sets the maximum evolution algebra $T$; the automatically generated $M$ individuals form the initial population.

Natural evolution follows the rule of survival of the fittest. In the genetic algorithm, in order to characterize the probability that each individual can obtain genetic information, an appropriate fitness function is selected as the criterion for judging the merits of individuals (solutions). The fitness function is directly proportional to the fitness of the individual. In different problems, the fitness function used is different. The selection criteria include standardization (which can include single value, continuous, and monotonous), rationality, and universality. In dealing with specific problems, the fitness function of individuals in the group can be obtained by calculation. In this paper, the objective function in the mathematical model is used to evaluate the pros and cons of a chromosome to find the optimal solution.

The selection operation is one of the most basic operations in the genetic algorithm. In the genetic algorithm, the fitness function is used as an indicator, and individuals with high fitness are selected as the next generation to prepare for the next genetic evolution operation. Generally, individuals with greater fitness are more likely to be selected as the next generation. Commonly used selection operators are fitness ratio method, best individual preservation method, expected value method, ranking method, and league selection method. The most commonly used method is the fitness ratio method, also called roulette or Monte Carlo selection method.

Assuming that the population size is $n$ and the fitness of the individual value $i$ is $f_{i}$, the probability of the $i$-th individual being selected as the next generation in the entire population is $p_{s i}$, and the relationship between them is 


$$
p_{s i}=\frac{f_{i}}{\sum_{i=0}^{M-1} f_{i}} .
$$

The selection probability $p_{s i}$ indicates that the greater the fitness of an individual in the entire population, the higher the probability that it will be selected as the next generation. Conversely, the smaller the fitness, the higher the probability of being eliminated.

Through selection operations, some excellent individuals can be selected from the current population, so that the selected individuals have the opportunity to become parents, reproduce offspring, and inherit some excellent individuals from the previous generation according to specific rules. Due to the different fitness of individuals, the fitness of offspring will be different. Individuals with high fitness are selected and so on until the end of the selection.

In the genetic algorithm, the crossover operation is used to simulate the genetic recombination in the biological evolution process and is the most important operation. For the two selected chromosomes, randomly we select their crossover points for crossover and exchange information with each other to form new offspring chromosomes. The crossover operation does not use individual fitness information, so it can keep the population mean unchanged. The crossover operation is very important in genetic algorithms. On the one hand, it allows excellent individuals in the original population to be maintained. It enables the algorithm to search for new gene spaces, which can make the algorithm jump out of the local optimal solution to a certain extent.

In the process of biological evolution, biological traits will change as the living environment changes. For genetic algorithms, mutation operation is to imitate the mutation in biological evolution, select an individual from the population arbitrarily, and then change the chromosome information of that individual with a certain probability. Mutation can make the genetic algorithm have local random search capabilities. The use of mutation operation can accelerate the population to converge to the optimal solution; mutation can maintain the diversity of the population, which can prevent the algorithm from falling into the local optimal solution to a certain extent, avoid premature phenomena, and improve the solving ability of genetic algorithm. The flowchart of genetic algorithm is shown in Figure 4.

\section{Simulation Experiment and Analysis}

4.1. Variable Load Dispatch Experiment in Static Control System. In the simulation experiment, the time range is set from $21: 00$ on the first day to $21: 00$ on the second day, and the load scheduling interval is set to 1 hour; then $T=24$. We select 100 units to participate in the experiment, assuming that there are $N$ rechargeable electric vehicles that can be used for dispatch.

The load data of the nonadjustable load comes from the load value in 2019. In the experiment, the load value at each moment is taken as the average value of that moment every day in 2019, as shown in Figure 5.
Renewable energy power generation data comes from the total wind power generation data recorded by a provincial power system operator every ten minutes from 2015 to 2019. Since wind power generation is greatly affected by seasons and weather, the wind power generation curves of four typical dates are selected to represent four different seasons. The simulation results are shown in Figure 6.

In addition, in the simulation experiment of this article, $N$ adjustable loads are set to be connected to the grid at the starting time (21:00 on the first day) and are in a dispatchable state. In the experiment, a rechargeable electric vehicle was selected as the representative of adjustable load. Assuming that $N$ rechargeable electric vehicles are exactly the same, the total electricity demand of each electric vehicle in the experiment is $12 \mathrm{KWh}$, and the charging rate is between $[0,3.1] \mathrm{KW}$.

It is worth noting that the value of the total adjustable load $N$ will also have a certain impact on the scheduling result. Normally, it is reasonable to select an adjustable load value of $5 \%-30 \%$ of the total load. Correspondingly, in the simulation experiment, the number of rechargeable electric vehicles is set to be 50 for research, and the optimality of the traditional mixed-integer programming load scheduling algorithm and the genetic optimization algorithm is compared, and the simulation results are shown in Figure 7.

Figure 7 is the result of scheduling using genetic optimization algorithm and mixed-integer programming when the total adjustable load $N$ is 50 . It can be seen from Figure 7 (a) that the total load curve obtained by the genetic optimization algorithm is closer to the change of the power supply curve. Comparing the curves of the absolute difference between the planned power supply and the total load value obtained by the two algorithms in Figure 7(b), it can be seen that the curve obtained by the mixed-integer planning and dispatching has large fluctuations.

\subsection{Adjustable Load Optimization Experiment in Real-Time} Distributed System. The mean square prediction error value is only related to the prediction moment. Figure 8 shows the error curve of standardized wind forecast within 24 hours. It can be seen from the figure that there are 6 peaks in the predicted value at several predicted moments.

In the simulation, the rechargeable electric vehicle is selected to represent the adjustable load, and the electricity consumption schedule of the adjustable load is the optimal charging curve of the electric vehicle. The simulation uses the relevant data of electric vehicles and assumes that the relevant parameters of all electric vehicles are exactly the same; that is, the total power demand of each electric vehicle is $12 \mathrm{kWh}$, and the rated charging power is between $[0,3.1] \mathrm{k}$ $\mathrm{W}$. The difference from the static control system simulation experiment is that the charging time of the regulated load connected to the grid starts at $21: 00$ on the first night and ends at 12:00 on the second day. After the deadline, all electric vehicles have completed charging.

At time $t$, the genetic optimization algorithm scheduling algorithm only dispatches $N(t)$ adjustable loads that have been connected to the grid, so that the total load 


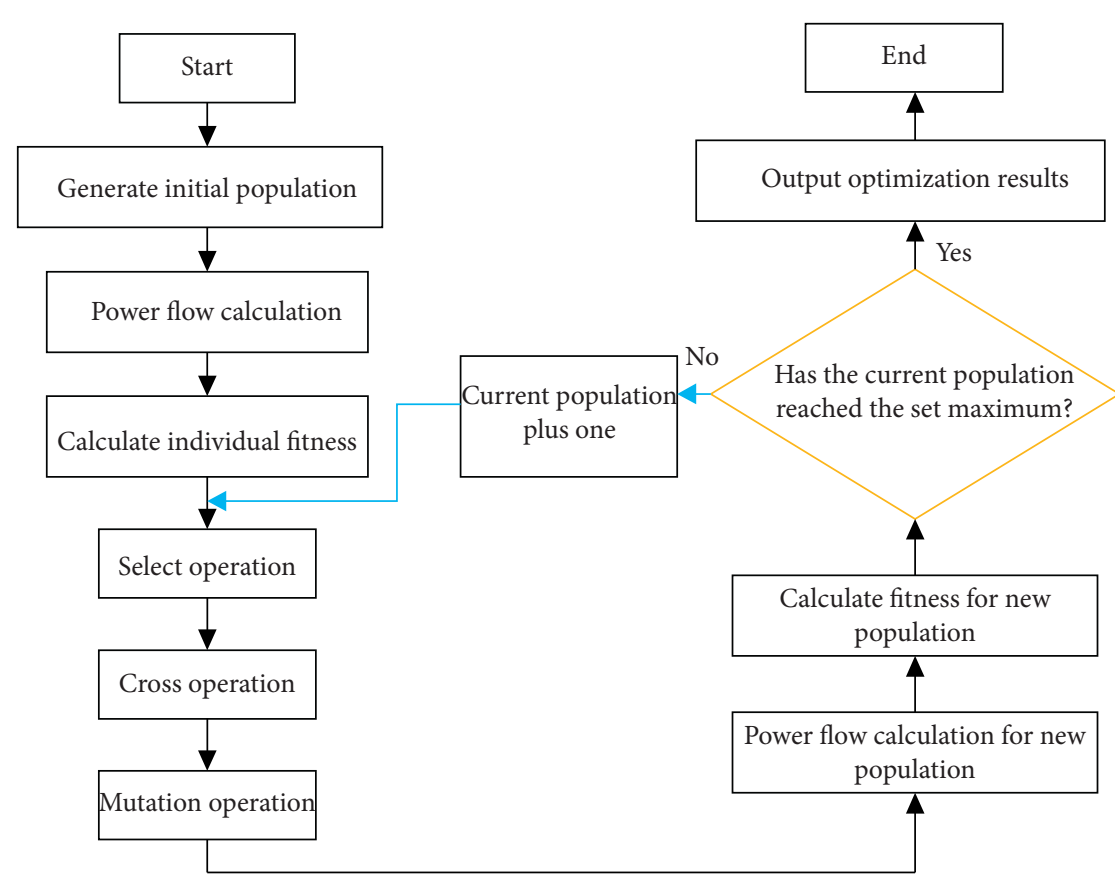

FIgURE 4: The solution process of genetic algorithm.

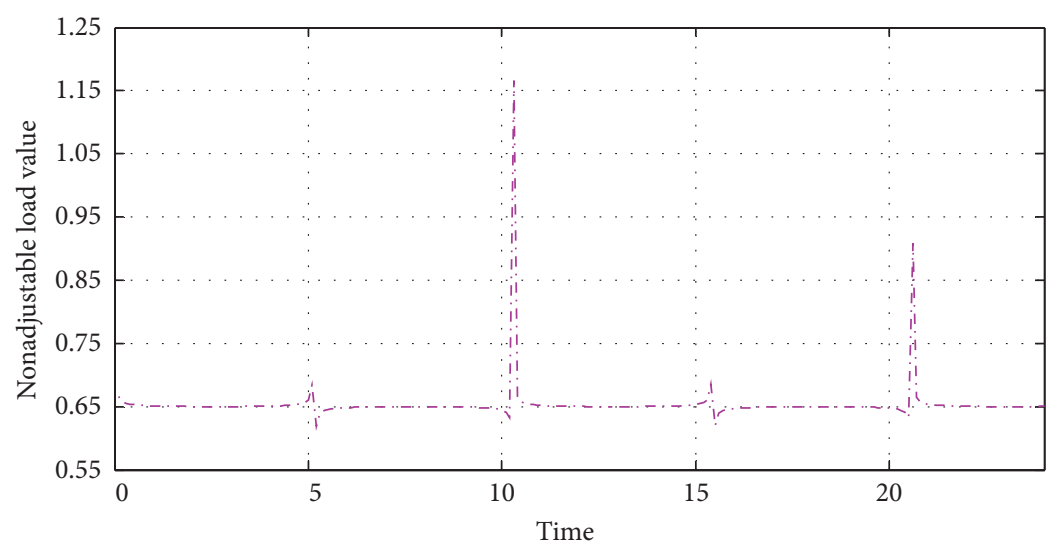

Figure 5: Nonadjustable load value.

power curve is as close to the power supply curve as possible, and the power resources provided by the power market can be maximized. The simulation obtains the power consumption schedule of the adjustable load at each moment and compares the total load power curve obtained by the mixed-integer planning scheduling algorithm and the genetic optimization algorithm scheduling algorithm with the power supply curve. Figure 9 selects the comparison result at $t=12$.

Figure 9(a) shows the comparison result of the total load curve of the two algorithms for load scheduling at different times. It can be seen from the above simulation results that because the mixed-integer programming scheduling algorithm only takes the reduction of the peak-valley difference of the load curve as the target strategy for load scheduling, it is not restricted by the power supply curve. The total load power value obtained by the mixed-integer planning scheduling algorithm for load scheduling exceeds the maximum planned power supply, which will cause the power market to exceed supply, and at the same time, it will have a certain impact on the stability of the power system. In addition, it can be seen from the simulation diagram that when using genetic optimization algorithm for real-time control, except for a few individual points, the total load curve is always below the power supply curve, effectively avoiding the situation where the total load power is greater than the supply value. Because the algorithm itself contains power supply constraints, it can be seen from the figure that, compared to the scheduling results of the mixed-integer programming scheduling algorithm, the total load curve obtained by the genetic optimization algorithm is closer to the power supply curve.

Figure 9(b) shows the absolute difference curve between the planned power supply and the total load power obtained 


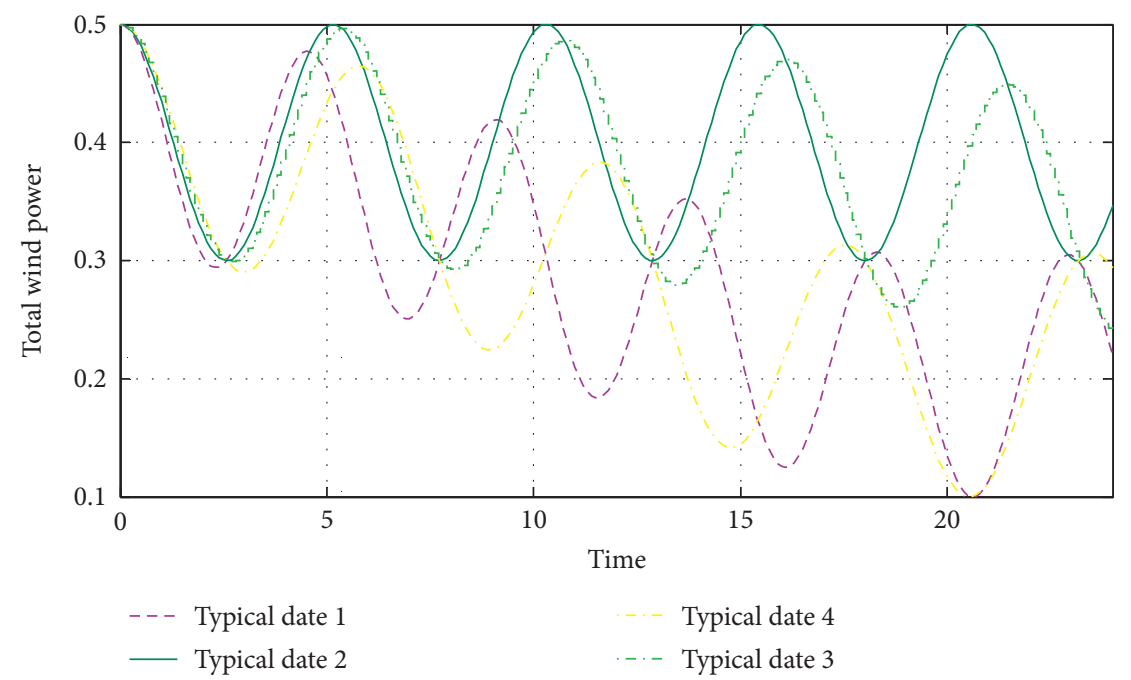

FIgURE 6: Total wind power generation.

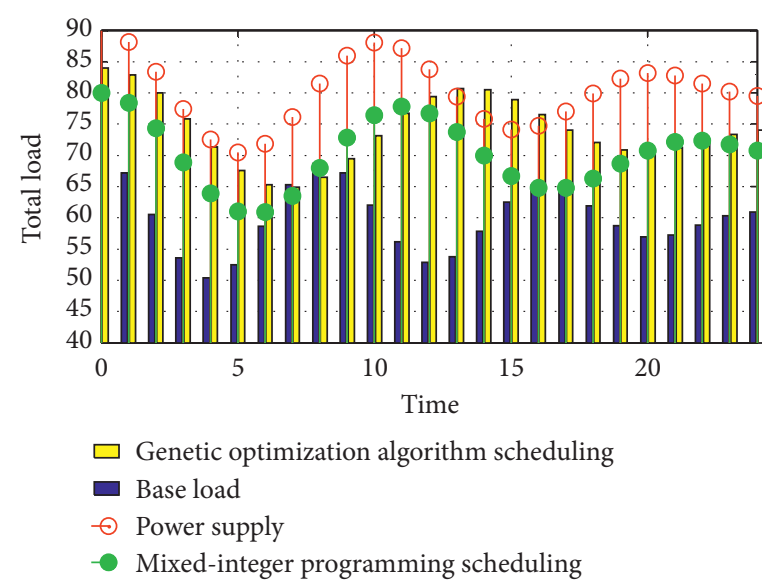

(a)

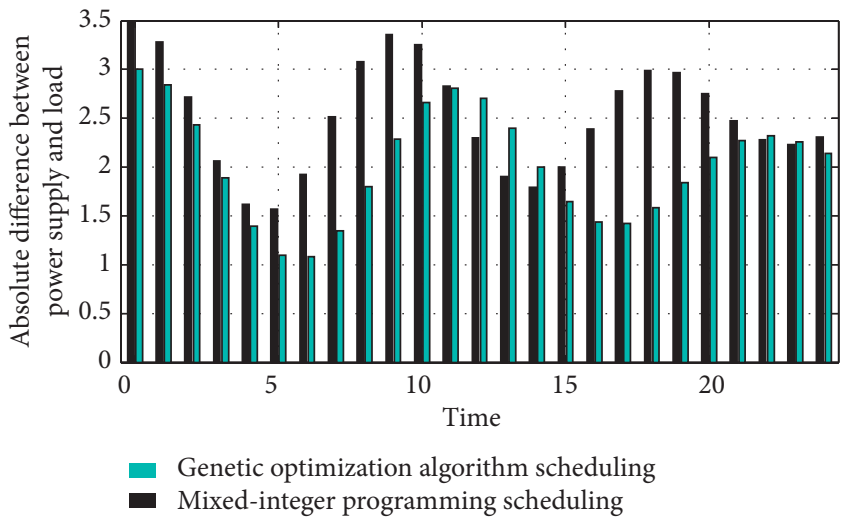

(b)

FIGURE 7: Comparison of total load and remaining unused power obtained by the two algorithms when $N=50$. (a) Load power curve comparison. (b) Comparison of absolute difference between power supply and load.

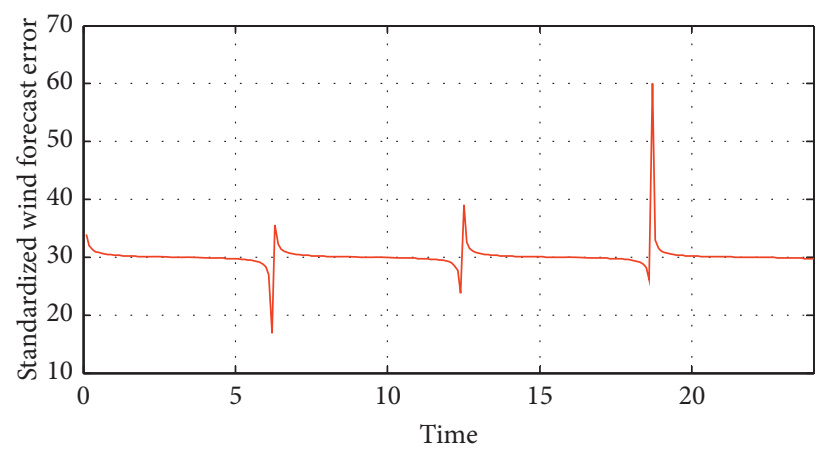

Figure 8: Wind forecast error with standardized mean square value. 


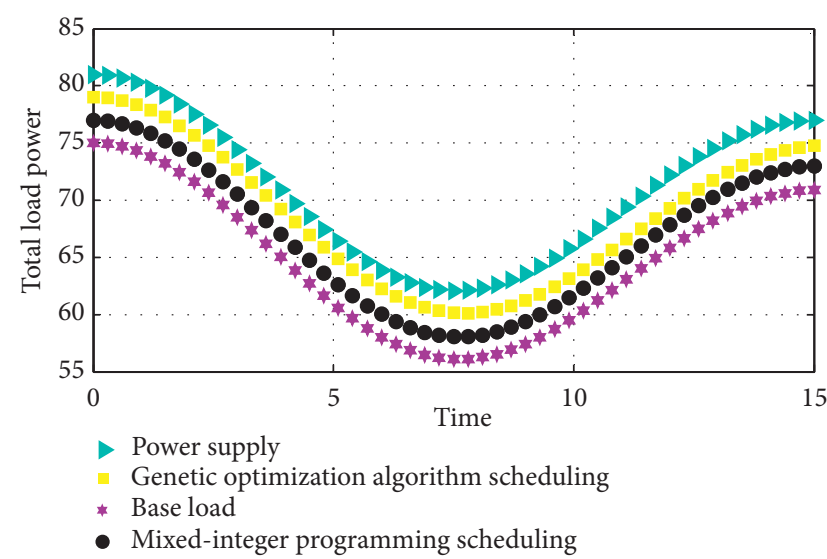

(a)

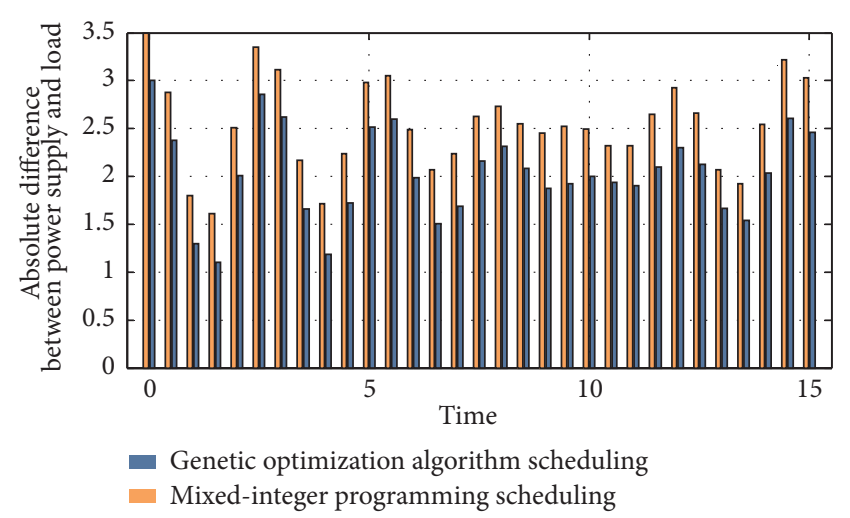

(b)

Figure 9: Comparison of two algorithms at $t=12$ in real-time distributed system. (a) Load power curve. (b) Comparison of absolute difference between power supply and load.

by the two algorithms for load scheduling. It can be seen from the above simulation diagram that, compared to the mixed-integer programming and scheduling algorithm, the difference obtained by the genetic optimization algorithm is generally maintained at a relatively low level. At this time, the power supply resources provided by the power market can be more fully utilized, and the remaining unused power is less, effectively avoiding the waste of resources.

\section{Conclusion}

Based on the analysis of power generation plan and maintenance plan optimization model, this paper establishes a smart grid power generation and maintenance collaborative optimization model with distributed renewable energy. The objective function of the collaborative optimization problem is the operating cost of conventional units and the cost of wind power generation. The constraints considered mainly include two parts: system constraints (power balance constraints, spinning reserve constraints, unit output upper and lower limit constraints, and unit climbing constraints) and maintenance constraints (maintenance status constraints, maintenance time constraints, maintenance continuity constraints, mutually exclusive maintenance constraints, and the relevance constraints between the maintenance status and the start-stop status). It analyzes the solution method of combinatorial optimization and selects and introduces the genetic optimization algorithm adopted in this paper. The simulation experiment with MATLAB software verifies the effectiveness of this algorithm. This algorithm overcomes the limitations of traditional algorithms that only satisfy valley filling and does not consider power supply constraints, so it is more practical and can ensure the full use of power resources and also help maintain the relative stability of the power system. Under the condition of power supply constraints, the genetic optimization algorithm can achieve the total load curve approaching the power supply curve. In the simulation experiment, the effectiveness of the genetic optimization algorithm was further verified by comparing it with the traditional load scheduling algorithm (mixed-integer programming scheduling algorithm). Since the mixed-integer programming and scheduling algorithm is only used under ideal conditions without power supply constraints, the genetic optimization algorithm overcomes this limitation and is more in line with the actual situation. When the genetic optimization algorithm is used for load real-time dispatch, the power supply resources of the power market can be fully utilized, which is beneficial to the safe and effective operation of the power system.

\section{Data Availability}

The data used to support the findings of this study are available from the corresponding author upon request.

\section{Conflicts of Interest}

The authors declare that they have no known competing financial interest or personal relationships that could have appeared to influence the work reported in this paper.

\section{References}

[1] S. Singh, V. B. Pamshetti, and S. P. Singh, "Time horizonbased model predictive volt/VAR optimization for smart grid enabled CVR in the presence of electric vehicle charging loads," IEEE Transactions on Industry Applications, vol. 55, no. 6, pp. 5502-5513, 2019.

[2] C. Xiao, D. Sutanto, K. M. Muttaqi, and M. Zhang, "A judicious decision-making approach for power dispatch in smart grid using a multiobjective evolutionary algorithm based on decomposition," IEEE Transactions on Industry Applications, vol. 56, no. 2, pp. 1918-1929, 2019.

[3] Y. Du, J. Wu, S. Li, C. Long, and S. Onori, "Coordinated energy dispatch of autonomous microgrids with distributed MPC optimization," IEEE Transactions on Industrial Informatics, vol. 15, no. 9, pp. 5289-5298, 2019.

[4] X. He, Y. Zhao, and T. Huang, "Optimizing the dynamic economic dispatch problem by the distributed consensusbased ADMM approach," IEEE Transactions on Industrial Informatics, vol. 16, no. 5, pp. 3210-3221, 2019. 
[5] W. Yin, D. Mavaluru, M. Ahmed, M. Abbas, and A. Darvishan, "Application of new multi-objective optimization algorithm for EV scheduling in smart grid through the uncertainties," Journal of Ambient Intelligence and Humanized Computing, vol. 11, no. 5, pp. 2071-2103, 2020.

[6] A. H. Bagdadee and L. Zhang, "Smart grid implementation of the industrial sector," International Journal of Energy Optimization and Engineering, vol. 8, no. 4, pp. 1-14, 2019.

[7] R. Lin, Z. Ye, and B. Wu, "The application of hydrogen and photovoltaic for reactive power optimization," International Journal of Hydrogen Energy, vol. 45, no. 17, pp. 10280-10291, 2020.

[8] H. Guo, F. Wang, L. Zhang, and J. Luo, "A hierarchical optimization strategy of the energy router-based energy internet," IEEE Transactions on Power Systems, vol. 34, no. 6, pp. 4177-4185, 2019.

[9] B. Wang, P. Dehghanian, and D. Zhao, "Chance-constrained energy management system for power grids with high proliferation of renewables and electric vehicles," IEEE Transactions on Smart Grid, vol. 11, no. 3, pp. 2324-2336, 2019.

[10] R. E. Martínez, E. C. Bravo, W. A. Morales, and J. D. GarciaRacines, "A bi-level multi-objective optimization model for the planning, design and operation of smart grid projects. Case study: an islanded microgrid," International Journal of Energy Economics and Policy, vol. 10, no. 4, pp. 325-341, 2020.

[11] Y. Yao, F. Tian, F. Mei, J. Fu, B. Dai, and W. Yu, "Dynamical economic dispatch using distributed barrier function-based optimization algorithm," Science China Technological Sciences, vol. 62, no. 12, pp. 2104-2112, 2019.

[12] S. Lei, C. Chen, Y. Li, and Y. Hou, "Resilient disaster recovery logistics of distribution systems: co-optimize service restoration with repair crew and mobile power source dispatch," IEEE Transactions on Smart Grid, vol. 10, no. 6, pp. 61876202, 2019.

[13] T. S. Mahmoud, B. S. Ahmed, and M. Y. Hassan, "The role of intelligent generation control algorithms in optimizing battery energy storage systems size in microgrids: a case study from Western Australia," Energy Conversion and Management, vol. 196, pp. 1335-1352, 2019.

[14] X. Wu, Y. Sun, Z. Wei, and G. Sun, "Distributed hierarchical consensus algorithm for economic dispatch in smart grid," IET Generation, Transmission \& Distribution, vol. 13, no. 24, pp. 5541-5549, 2019.

[15] M. A. Velasquez, J. Barreiro-Gomez, N. Quijano et al., "Intrahour microgrid economic dispatch based on model predictive control," IEEE Transactions on Smart Grid, vol. 11, no. 3, pp. 1968-1979, 2019.

[16] T. Liu, D. Zhang, H. Dai, and T. Wu, "Intelligent modeling and optimization for smart energy hub," IEEE Transactions on Industrial Electronics, vol. 66, no. 12, pp. 9898-9908, 2019.

[17] H. Liu, Y. Wang, M. Wang et al., "Small-signal analysis of DC microgrid and multi-objective optimization segmented droop control suitable for economic dispatch," Journal of Modern Power Systems and Clean Energy, vol. 8, no. 3, pp. 564-572, 2020.

[18] Y. Duan and X. He, "A non-convex dispatch problem with generator constraints using neural network and particle swarm optimization," Iranian Journal of Science and Technology, Transactions of Electrical Engineering, vol. 44, no. 1, pp. 185-196, 2020.

[19] K. Vankadara and I. Raglend, "A review to economic dispatch of hybrid microgrids," Serbian Journal of Electrical Engineering, vol. 16, no. 2, pp. 233-246, 2019.
[20] V. J. Gutierrez-Martinez, E. A. Zamora-Cardenas, A. PizanoMartinez, J. M. Lozano-Garcia, and M. A. Gomez-Martinez, "Optimal dispatch model for demand response aggregators," Journal of Electrical Engineering \& Technology, vol. 14, no. 1, pp. 85-96, 2019. 